Relations industrielles

Industrial Relations

\title{
Line, staff et functional
}

\section{Raymond Pepermans}

Volume 43, numéro 1, 1988

URI : https://id.erudit.org/iderudit/050395ar

DOI : https://doi.org/10.7202/050395ar

Aller au sommaire du numéro

\section{Éditeur(s)}

Département des relations industrielles de l'Université Laval

ISSN

0034-379X (imprimé)

1703-8138 (numérique)

Découvrir la revue

Citer cet article

Pepermans, R. (1988). Line, staff et functional. Relations industrielles / Industrial

Relations, 43(1), 183-187. https://doi.org/10.7202/050395ar
Résumé de l'article

Line, staff and functional
Tous droits réservés (C Département des relations industrielles de l'Universite Laval, 1988
Ce document est protégé par la loi sur le droit d'auteur. L'utilisation des services d'Érudit (y compris la reproduction) est assujettie à sa politique d'utilisation que vous pouvez consulter en ligne.

https://apropos.erudit.org/fr/usagers/politique-dutilisation/ 


\title{
DISCUSSION
}

\section{Line, staff et functional}

\author{
Raymond Pepermans
}

La traduction de ces termes en langue française a toujours posé des problèmes aux traducteurs oeuvrant dans le domaine de la gestion et de l'administration. Cette situation reflète les profondes divergences qui existent entre les spécialistes des sciences de l'organisation au sujet de ces concepts. Cependant, on constate que cette complexité, à laquelle font allusion la plupart des auteurs ayant traité de ce sujet, est en partie exagérée lorsqu'on se penche d'abord sur la réalité organisationnelle dans sa matérialité, telle qu'elle se présente sous nos yeux, en remettant à plus tard toute considération d'ordre traductionnel.

Un certain nombre d'ouvrages récents nous permettent d'améliorer notre compréhension du sujet. Ces ouvrages nous présentent l'organisation comme étant composée d'un certain nombre de types de structures de complexité croissante, chaque type étant caractérisé par l'adjonction d'un principe structurel de nature différente. Voyons en quoi consistent ces trois structures qui ont été qualifiées en anglais de structures line, functional, staff and line.

\section{a) La structure line}

C'est en quelque sorte la «structure élémentaire», la plus ancienne, la plus simple et qui se définit selon le principe hiérarchique: chaque employé n'a qu'un seul supérieur et ce dernier a une autorité sans partage sur ses subordonnés. C'est aussi ce qu'on appelle en français le plus courant la structure hiérarchique.

\section{b) La structure functional}

Dans ce type de structure, un supérieur n'exerce une autorité définie que dans une spécialité déterminée, une fonction de l'entreprise et non sur

* PEPERMANS, Raymond, terminologue, division centrale, direction de la terminologie, bureau des traductions, Secrétariat d'État, Ottawa.

** La première version de ce texte parue dans l'Actualité terminologique, bulletin mensuel du Bureau de traduction du Secrétariat d'État du Canada, volume 20, no 1, 1987, qu'avec autorisation requise nous reproduisons ici, a été complétée par l'auteur qui y a ajouté des additions. 
l'ensemble des opérations effectuées par chaque service comme dans la structure hiérarchique. Chaque employé relève de plusieurs supérieurs, par exemple pour le réglage et la manutention, l'entretien, le rendement et les instructions, le contrôle de la qualité, la paye, la discipline, etc... tandis que le cadre n'exerce sa compétence que dans un seul domaine mais, cette fois, au niveau de toute l'entreprise. Cette structure, qu'on a souvent confondue avec la structure line and staff, est plus complexe que la structure hiérarchique dans la mesure où le principe fonctionnel n'est applicable qu'aux échelons inférieurs de l'entreprise. Il est donc nécessaire de conserver le principe hiérarchique aux échelons supérieurs de l'organisation. Cependant, comme on caractérise toujours une structure par ses aspects essentiels, on appellera cette dernière la structure fonctionnelle.

\section{c) La structure staff and line}

Cette structure se caractérise par l'adjonction, au principe hiérarchique, d'un certain nombre de services et de personnes exerçant, dans un premier temps, des fonctions supplétives par rapport aux activités normales de l'entreprise. Ces fonctions finissent par devenir aussi essentielles à la marche de l'organisation que les services hiérarchisés, à la différence qu'elles ne sont pas intégrées à la structure line. Les services du personnel en fournissent un exemple frappant: d'abord assumée dans le cadre hiérarchique, la fonction «personnel» a été progressivement confiée à un petit nombre de spécialistes situés en retrait par rapport à celui-ci. Avec la complexité et la dimension grandissante des entreprises, ces services ont été obligés d'adopter eux-mêmes une structure line, tout en restant staff par rapport aux autres fonctions de l'organisation. L'élément staff est un élément structurel se présentant sous la forme d'une relation de conseil vis-à-vis des autres composantes de l'entreprise, alors que la structure hiérarchique repose sur la relation de commandement en ligne directe. Il existe plusieurs sortes de staff: le staff de consultation, qui est celui des spécialistes; le staff fonctionnel, s'exerçant dans une spécialité donnée; le staff personnel, qui est celui des comités; le staff général, auquel un cadre abandonne certains pouvoirs pour une période déterminée. La structure staff and line, dans laquelle on retrouve concurremment trois principes structurels hétérogènes: line, staff et functional, est appelée structure mixte ${ }^{1}$.

Comme nous venons de le voir, ces trois modes de structure ne présentent aucun problème insurmontable quant à leur intelligibilité. D'autre part, une fois qu'ils sont correctement compris, c'est-à-dire une fois que la structure fonctionnelle n'est pas confondue avec la structure mixte, on constate que la langue française ne manque d'aucune ressource pour les désigner.

Les éclaircissements que nous venons d'apporter au problème de la désignation des structures de l'organisation nous permettent d'élucider le problème de la désignation en français des termes formés à partir des éléments syntagmatiques line, staff, functional.

1 SAUVÉ, Madeleine, Line et staff, Fiche d'observations grammaticales et terminologiques, $\mathrm{n}^{\circ} 77$, avril 1977. 


\section{1) Line}

Nous retenons l'adjectif hiérarchique en parlant de la structure hiérarchique, des services hiérarchiques, des cadres hiérarchiques, etc... Les équivalents suivants traduisent mal la réalité exprimée par cette notion:

- services organiques: toute structure est organique en ce sens qu'elle fonctionne comme un ensemble d'organes. C'est surtout le cas de la structure fonctionnelle qui peut être comparée à un organisme vivant dans lequel chaque organe exerce un rôle.

- structure linéaire: toute structure est linéaire dans la mesure où elle peut être représentée par un organigramme composé de lignes joignant un certain nombre de cases schématisant l'ensemble des éléments de l'organisation. Ces éléments ne sont pas nécessairement hiérarchisés.

- services opérationnels, d'exécution, d'exploitation: ces adjectifs ne décrivent en rien la spécificité de cette notion (line). Ils renvoient à des activités qui peuvent être exercées aussi bien dans la structure hiérarchique que dans les structures fonctionnelle et d'état-major.

- services axiaux: cet équivalent, proposé par le Comité d'étude des termes techniques français, n'est pas incorrect, mais il est surprenant dans la mesure où il existe déjà un terme consacré pour caractériser cette notion. Depuis longtemps, dans le vocabulaire de la sociologie des organisations, on appelle cette réalité structure hiérarchique. L'adjectif axial n'apparaît nulle part dans les ouvrages classiques consacrés à ce domaine. Nous ne voyons pas l'intérêt d'ajouter ce néologisme inutile alors que l'adjectif hiérarchique est compris de tous. Tout le monde comprendra ce qu'est un cadre hiérarchique, personne ce que c'est qu'un cadre axial.

Nous croyons qu'il faut éviter de recourir à d'autres équivalents pour ce qui est des composés de line. Dans le cas où cela s'avère impossible, il faut alors recourir à un vocable qui évoque de près ou de loin l'idée de hiérarchie. Par exemple, pour line department ou line ministry, on traduira par ministère responsable (sous-entendu: ministère hiérarchiquement responsable).

\section{2) Staff}

Ce sont les termes consultatif, de consultation qui rendent le mieux cette notion. On peut aisément les utiliser en association avec les termes suivants: autorité, compétence, fonction, cadre, agent, etc... Ce n'est cependant pas toujours possible et il faudra recourir à l'expression d'étatmajor pour qualifier un service, des liaisons, etc... Ce terme, peu populaire auprès de nos clients francophones à cause de sa connotation militaire, est cependant consacré par l'usage dans le vocabulaire de la gestion. Non seulement il rend bien le terme anglais correspondant mais, d'autre part, il procède d'une extension de sens particulièrement heureuse. Dans son acception militaire, ce terme désigne un ensemble d'officiers sans troupes, donc en 
dehors de la structure line, chargés d'un ensemble de services techniques et d'un rôle consultatif auprès du commandement suprême. Cette notion a été étendue au domaine politique: on parle de l'état-major d'un ministre ou celui d'un parti politique. Rien ne s'oppose à ce qu'il y ait un état-major auprès de la direction générale d'une entreprise.

\section{3) Staff and line}

Comme nous l'avons vu ci-dessus, nous sommes d'accord avec Madame Sauvé pour retenir l'expression structure mixte, non seulement pour les raisons qu'elle invoque pour rejeter le terme «fonctionnel» comme équivalent pour staff, mais aussi pour ne pas confondre deux structures: le staff peut être fonctionnel mais il ne l'est pas toujours. Cette expression est difficilement traduisible de façon littérale en français parce que nous ne disposons pas, comme en anglais, de deux termes monosyllabiques commodes qu'il suffit de mettre en apposition.

\section{4) Functional}

Ce terme se traduit par fonctionnel, qu'il s'agisse d'une entité administrative (service fonctionnel), d'une personne (cadre fonctionnel) ou d'un rapport social (relation fonctionnelle). Il ne doit pas, bien entendu, être compris dans son acception courante qui renvoie à «ce qui fonctionne» ou à «ce qui contribue au fonctionnement de» mais à «ce qui est relatif à une fonction, un rôle» dans une structure sociale particulière. Contrairement à ce qu'affirme Madeleine Sauvé, ${ }^{2}$ ce vocable n'est pas ambigu; fonctionnel est, dans ce sens, très répandu dans les autres spécialités des sciences humaines. Nous ne voyons pas pourquoi la perspective fonctionnaliste devrait être rejetée dans les sciences de l'organisation, d'autant plus que, dans ces sciences, les modèles fonctionnels décrivant la réalité organisationnelle ne sont pas uniquement des modèles heuristiques, mais des représentations du réel.

Nous avons constaté que les difficultés posées par ces termes se ramènent à peu de choses, à condition d'avoir élucidé la distinction qui existe entre la structure fonctionnelle et la structure mixte. Les équivalents retenus s'avèrent peu nombreux. Ils épuisent complètement la réalité exprimée par ces notions mais, sous réserve, comme dans le cas de line department, d'explorer toutes les ressources de la traduction lorsque nécessité s'impose.

L'organisation la plus récente, représentant le niveau de complexité le plus élevé, est la «matrix organization ${ }^{3}$ ou organisation matricielle, aussi appelée organisation en matrice ${ }^{4}$. Ce type de structure ne représente aucune

2 SAUVE, Madeleine, op. cit.

3 BANKI, Ivan S., Dictionary of Administration and Management, Authoritative, Comprehensive, Los Angeles, California, Systems Research, 1974, p. 462.

4 Encyclopédie du management (Centre d'enseignement supérieur des affaires), rédacteur en chef, Jacques Lendrevie, Paris, France - Expansion, 1977, vol. I, 2.22, p. 11. 
difficulté sur le plan traductionnel comme l'attestent les deux équivalents susmentionnés. Cependant elle nécessite quelques éclaircissements à l'intention du lecteur intéressé à ce que recouvre cette notion.

Cette organisation doit être envisagée dans le cadre de la gestion de projets. Il s'agit d'une pratique administrative qui consiste à désigner soit des superviseurs «mixtes», participant conjointement aux structures «hiérarchique» et «consultative», comme nous l'avons mentionné ci-dessus ou des superviseurs "fonctionnels», soit des employés appartenant à ce genre d'organisation dans le but d'exercer leurs tâches au sein d'une multiplicité de fonctions relevant de cette même organisation. Il peut aussi s'agir de gestionnaires ou d'employés exerçant une même tâche à des niveaux de responsabilité différents en vue de réaliser les objectifs d'un programme à court, à moyen ou à long terme. L'organisation matricielle se caractérise ainsi par un recouvrement des trois structures, hiérarchique, d'état-major ou fonctionnelle avec adjonction d'un élément de flexibilité supplémentaire qui correspond à la complexité de plus en plus grande des organisations bureaucratiques contemporaines.

La matrice, terme emprunté aux mathématiques, est aussi conçue comme une représentation sous forme d'un tableau comportant des lignes et des colonnes $^{5}$ permettant de symboliser schématiquement l'ensemble des tâches, fonctions et affectations au sein d'une organisation en tenant compte de l'élément de souplesse caractéristique de ce type de structure.

L'organisation contemporaine, de par la complexité croissante de sa structure, pose des problèmes terminologiques non seulement aux spécialistes de la traduction et de la terminologie mais aussi aux praticiens de la gestion et aux sociologues des organisations. Nous apprécierions tout commentaire sur nos propos de la part des représentants de ces différentes disciplines.

5 Le Grand Robert de la langue française, Dictionnaire alphabétique et analogique de la langue française, Paris, $2^{\mathrm{e}}$ édition, revue et enrichie par Alain Rey, Tome VI, p. 308. 